\title{
EFFECTS OF AGGREGATION ON THE PROBABILITY OF DETECTING INFESTATIONS IN FRESH PRODUCE CONSIGNMENTS
}

\author{
M.C. BARRON \\ AgResearch, PO Box 60, Lincoln, New Zealand \\ Corresponding author: mandy.barron@agresearch.co.nz.
}

\begin{abstract}
Border inspections of imported fresh produce aim to detect pest or disease infestations of 0.005 prevalence with $95 \%$ confidence and, based on the binomial distribution, a sample of 600 produce units is typically inspected from each consignment. Depending on the type of produce, units are selected at either the individual or the carton level to make up the sample size. However, sampling cartons (cluster sampling) violates the assumption of the binomial distribution that the sampling units are independent of one another. This may be of no consequence if the pests or diseases are distributed randomly throughout the consignment, but aggregation of invertebrates and disease within a consignment is likely to occur. Simulation models are used to demonstrate how cluster sampling leads to a decreased probability of detecting infestations and an underestimate of the true level of infestation with increasing levels of aggregation.

Keywords: MAF biosecurity, binomial distribution, cluster sampling, simple random sampling.
\end{abstract}

\section{INTRODUCTION}

To detect infestations of pests and disease in imported fresh produce the default Ministry of Agriculture and Forestry (MAF) sampling protocol is to inspect 600 produce units from each consignment. This standard sample size is calculated from the binomial distribution, which predicts that given a large consignment with a 0.005 prevalence of infestation, then a sample size of 600 units will have a 0.95 probability of detecting that infestation. Conversely, this means that there is a 0.05 probability that the infestation will not be detected. However, predictions from the binomial distribution are based on the assumption that the prevalence of infestation is constant throughout the consignment (i.e. there is no aggregation) and that simple random sampling is used so that sampling observations are independent (Venette et al. 2002). In reality, natural aggregations of invertebrate pests and plant disease are likely to be maintained in produce consignments because the produce is typically packed into cartons. Also, MAF import health standards for the importation and clearance of fresh fruit and vegetables (MAF Biosecurity Authority 2004) are unclear as to what method of sampling is to be used:

"A sample shall be taken from each lot as specified in the appropriate sampling plan detailed below. Samples may be taken from any part of the lot and shall include a selection of cartons/packages from different areas of the lot. To ensure a representative sample is selected for inspection, consideration should be given to selecting cartons from different grower lines, different pallets, different brands and different locations within the consignment."

(Section 4.4: MAF Biosecurity Authority 2004)

The above excerpt implies that the carton is the sampling unit, which would indicate cluster sampling, and in practice, cluster sampling is usually used because sampling the entire carton of produce allows the inspector to also check the packaging for signs of pest or disease infestation (G. Edwards, pers. comm.). 
For some commodities, there is a maximum allowable prevalence (MAP), which is the maximum level of infestation based on inspection, below which phytosanitary actions do not have to be applied. For fresh produce the MAP is 0.005 , which, given a 95\% confidence interval, means that zero units of a 600 unit sample are allowed to be infested (MAF Biosecurity Authority 2004). However, if a higher MAP was set for certain commodities, then the accuracy of the prevalence estimated from the sample becomes important. In particular the negative bias of prevalence estimates, or how often the prevalence estimated from the sample is lower than the true consignment prevalence, will determine if the MAP criteria is correctly applied.

The aim of this work was to investigate, using simulation models, the effects of disease or pest aggregation and the effects of different sampling methods on the probability of detecting infestations. An example scenario of sampling bananas for pests and diseases is used but the general results apply to any other type of produce.

\section{METHODS}

Each "hand" or bunch of bananas is classed as one produce unit and they are typically packaged in cartons of approximately 16 units per carton. MAF interception records for 2004 (S.Wedde, unpubl. data) show that a consignment of bananas is composed of, on average, 360,000 units (rounded to nearest 100 units), which dividing by 16 gives an average of 22,500 cartons per consignment. For the model simulations, four levels of disease prevalence were tested, $p=0.005,0.01,0.03$ or 0.05 . The actual prevalence of infestation recorded from banana interception records in 2004 averaged 0.02 , which is within the range of simulated prevalence. The minimum prevalence simulated, $p=0.005$, is the minimum level of infestation that the standard 600 unit sample is designed to detect.

A range of aggregation levels was used in the simulations since no data on the actual levels in banana consignments were available. To capture the effects of aggregation in the simulation model, infested units were randomly assigned to a defined proportion of the consignment. If there was no aggregation within the consignment, infested units were randomly assigned to the whole of the consignment. With increasing aggregation, infested units were randomly assigned to a decreasing proportion of the consignment $(1.0,0.5$, 0.1 or 0.05 ). For ease of interpretation, an index of aggregation (IA) was calculated as the inverse of the proportion of the consignment that was allocated infested units, giving $I A=1,2,10$ or 20 . The $I A$ can be thought of as a constant (multiplier) for the prevalence in the infested part of the consignment. Thus if $p=0.005$ and there is no aggregation, so that infested units are throughout the consignment, then the effective prevalence is $0.005^{*} 1=0.005$, but if the infestation is concentrated in $1 / 20$ of the consignment then $I A=20$ and the effective prevalence in the infested part of the consignment is $0.005 * 20=0.1$.

Sampling of the consignment was simulated in two ways, simple random sampling, where the sampling unit was one unit of bananas, and cluster sampling, where the sampling unit was one carton of bananas (Fig. 1). Simple random sampling was simulated by randomly drawing 600 single unit samples from the consignment. Cluster sampling was simulated by randomly drawing 38 cartons from the consignment and sampling all 16 units within these cartons, except for the last carton drawn, in which only eight units were sampled so that the total number of units sampled summed to 600. For the model simulations, the appropriate number of infested units depended on the prevalence being simulated, and they were randomly allocated to a proportion of the 360,000 unit consignment, depending on the level of aggregation being simulated. A 600 unit sample was drawn using either simple random or cluster sampling and 100,000 iterations were run for each of these prevalence/aggregation/sampling method combinations. For each combination, the number of iterations in which no infested units were detected in the 600 unit sample were tallied. Dividing these tallies by the total number of iterations $(100,000)$ gives the proportion of infestations not detected (i.e. the proportion of false negatives). 
Similarly, a tally of the number of iterations where the prevalence estimated from the 600 unit sample was less than the lower 95\% confidence limit of the true prevalence was used to calculate the proportion of prevalence estimates that were negatively biased. The theoretical variance of the prevalence $s_{p}^{2}$ of a random $N$ unit sample where the true prevalence equals $p$ was calculated as: $s_{p}^{2}=p(1-p) / N-1$, giving an approximate $95 \%$ confidence interval of $p \pm\left(s_{p} * 2\right)$.

Aggregated consignment with infested individuals randomly allocated to half of the consignment $(I A=2)$
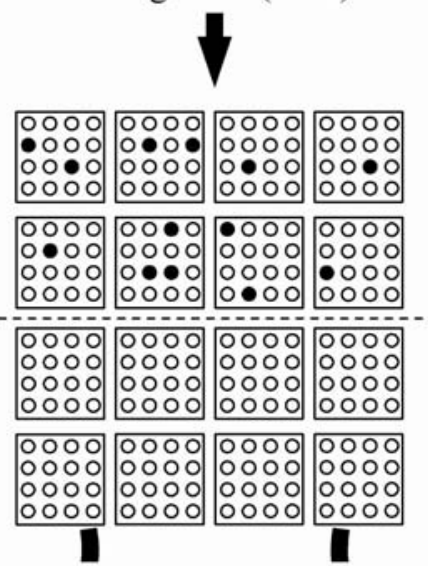

Simple random sampling Cluster sampling
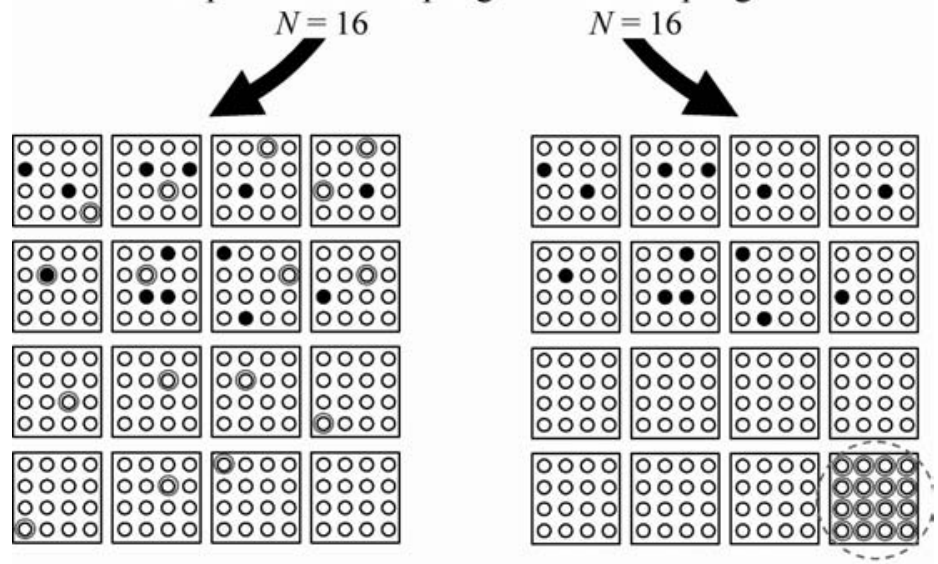

- = infested unit $\quad \mathrm{O}=$ unit sampled

FIGURE 1: Diagram showing model allocation of infested units in an aggregated consignment and the difference between simple random and cluster sampling. 


\section{RESULTS}

As expected, simulation of simple random sampling gave probabilities of missing an infestation (Fig. 2a) that were approximately the same as those calculated from the binomial distribution formula. There was also minimal difference in the proportion of prevalence estimates that were negatively biased under different levels of aggregation (Fig. 2c). Using cluster sampling, the degree of aggregation had a large effect on the likelihood of detecting an infestation and on the prevalence estimates being biased. For a prevalence of 0.005 , the probability of not detecting an infestation rose from 0.05 when there was no aggregation to 0.21 under high levels of aggregation (Fig. 2b). Even at a relatively high prevalence of 0.03 or 0.05 , the probability of making a false negative error was large, up to 0.14, under the highest level of aggregation simulated (Fig. 2b). The probability of the prevalence estimated from the sample being an under-estimate also rose with increasing aggregation (Fig. 2d). The proportion of estimates that were negatively biased increased with increasing prevalence, so that estimates from the highest $(p=0.05)$ prevalence simulations were the most biased under high levels of aggregation (Fig. 2d).
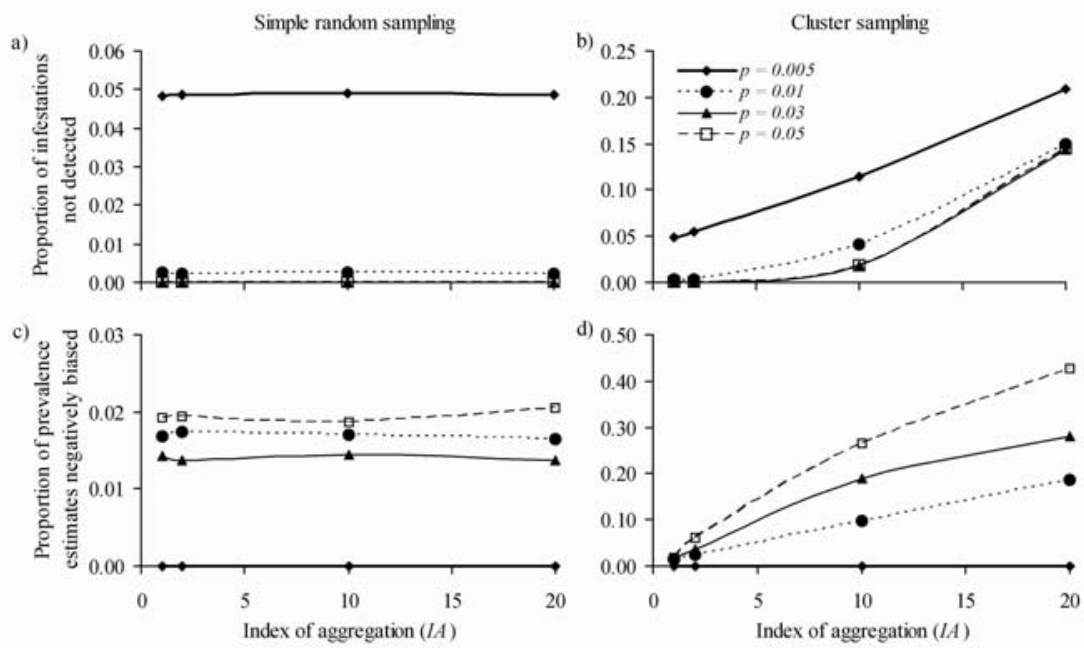

FIGURE 2: The proportion of infestations not detected by a 600 unit sample (a \& b), and the proportion of prevalence estimates from a 600 unit sample that were less than the lower $95 \%$ confidence limit of the true prevalence $(p)(c \& d)$, from simulations of simple random and cluster sampling under different levels of aggregation.

\section{DISCUSSION}

The decreased sensitivity of the cluster sampling method to disease/pest aggregation compared to random sampling, is worrisome because this is the sampling method typically used for produce inspections, resulting in much reduced ability to detect aggregated infestations. If there is pest/disease aggregation within a consignment and MAF's target of a $95 \%$ probability of detecting a 0.005 prevalence infestation is to be met, either simple random sampling must be adhered to or the number of clusters sampled must be increased. For low prevalence infestations $(p<0.1)$, the negative- 
binomial distribution can be used to calculate the requisite sample sizes for a given level of aggregation, prevalence, cluster size and probability of detection (Madden \& Hughes 1995; Venette et al. 2002). Under cluster sampling, the probability of under-estimating prevalence also rose dramatically with increasing aggregation, which could result in some consignments avoiding phytosanitary treatments, which under MAP criteria they would in fact require.

Levels of disease/pest aggregation within harvested produce have been little studied, so there are few data available to provide context. A search of the bibliographic database Scopus (www.scopus.com using search terms: sampling AND aggregation AND (prevalence OR incidence); searched in all subject areas from date range "before 1960-present" on 30 April 2005) revealed only two publications quantifying such aggregation (Marois et al. 1993; Copes et al. 2001). One of these looked at the prevalence of infection of rabbiteye blueberry fruit by mummy berry disease and found high levels of aggregation in some blueberry lots sampled and a positive relationship between levels of aggregation and disease prevalence (Copes et al. 2001). That study used different indices of aggregation to the $I A$ used here but calculation of the design effect ratio (deff = actual variance of sample divided by the predicted variance if there was no aggregation; Hughes et al. 1996) allows some comparison. For the blueberry data, samples where the prevalence was 0.005 or less had an average $d e f f=1.24$. This is approximately equivalent to $I A=4$ when $p=0.005$. If cluster sampling was used on blueberry lots with this level of prevalence and aggregation then there would be a 0.07 probability of not detecting an infestation compared with a 0.05 probability if single random sampling was used.

For operational purposes, it is difficult to provide recommendations for dealing with aggregations of disease/pest infestation in produce consignments, when the true levels of aggregation are unknown. Further, levels of aggregation will likely vary with the type of produce, the type of pest or disease, the disease/pest prevalence, the type of packaging and the country of origin. The simplest suggestion that can be offered to policy and quarantine personnel is to ensure that simple random sampling is adhered to as, unlike cluster sampling, this method is not affected by aggregation when inspecting for low levels of disease. To this end, the import health standards need to be clarified so that the unit of produce is unambiguously identified as the sampling unit. A diagram, similar to Figure 1, which illustrates how prone carton sampling is to missing infestations compared with single random sampling, might also be included.

Whether adherence to simple random sampling is an acceptable solution will depend on the commodity in question. For packed produce, simple random sampling is feasible, although the need to inspect the packaging as well as the produce could make cluster sampling a more practical choice. If this was the case, then it would be prudent to quantify the level of aggregation within these produce consignments to determine if it was large enough to affect the probabilities of detection and warrant an increased sample size to compensate. The cluster sampling that is typically used for produce inspections could provide information about levels of disease aggregation if the number of infested units per cluster (carton) is recorded, rather than just the total per 600 unit sample. For other commodity types, the statistical assumptions on which sampling schemes are based will invariably be violated because they are not practical to implement. For example, when sampling a seed consignment it would be unrealistic to expect quarantine personnel to take 600 random samples of individual seeds and some form of cluster sampling would probably be done instead. In these cases, other solutions for dealing with aggregation may have to be found, such as making sure seed consignments are well mixed so that any disease/pest aggregations within the consignment are broken up, making a cluster sample more representative of the total consignment. 


\section{ACKNOWLEDGEMENTS}

Thanks to Gavin Edwards and Sarah Wedde of Biosecurity New Zealand for supplying data and information. John Kean and Nigel Bell (AgResearch) provided useful comments on the manuscript. This work was funded by the FRST Better Border Biosecurity programme.

\section{REFERENCES}

Copes WE, Scherm H, Ware GO 2001. Sequential sampling to assess the incidence of infection by Monilinia vaccinii-corymbosi in mechanically harvested rabbiteye blueberry fruit. Phytopathology 91: 348-353.

Hughes G, Madden LV, Munkvold GP 1996. Cluster sampling for disease incidence data. Phytopathology 86: 132-137.

Madden LV, Hughes G 1995. Plant disease incidence: distributions, heterogeneity, and temporal analysis. Annual Review of Phytopathology 33: 529-564.

Marois JJ, Bledsoe AM, Ricker RW, Bostock RM 1993. Sampling for Botrytis cinerea in harvested grape berries. American Journal of Enology and Viticulture 44: 261-265.

Ministry of Agriculture and Forestry Biosecurity Authority 2004. MAF biosecurity authority (plants) standard 152.02: importation and clearance of fresh fruit and vegetables into New Zealand. Ministry of Agriculture and Forestry, Wellington, New Zealand. Report No. 152.02. 387 pp.

Venette RC, Moon RD, Hutchinson WD 2002. Strategies and statistics of sampling for rare individuals. Annual Review of Entomology 47: 143-174. 